\title{
DIE TCM - EINE SCHATZTRUHE MIT NOBELPREIS GEWÜRDIGT
}

\section{Liebe Kolleginnen und Kollegen,}

das Jahr neigt sich bereits dem Ende entgegen. Wir blicken zurück auf ein reichhaltiges Kursprogramm mit einer gut besuchten Grundausbildungslinie für Ärzte und Zahnärzte und interessanten Fortbildungskursen für Akupunkturärzte. In diesem Jahr wurden Kurse zur Liquidakupunktur und zur Punktstimulation neu ins Programm aufgenommen und erfreuten sich großer Beliebtheit.

Das Expertenseminar in Timmendorf fand erstmalig unter der Leitung der DAA e.V. statt. Das Vortragsprogramm wie auch die einzelnen Workshops waren vielgestaltig und erfreuten sich großen Zuspruchs. Allein 60 Teilnehmer nahmen am integrierten TCM Kurs 9 „Qi Gong“ teil. Energie tanken und die RAC-Fähigkeiten verbessern, Wissen aktualisieren und kollegialen Austausch pflegen: das waren die Ziele für diese Woche. Wie uns die Rückmeldungen bestätigten, konnten wir diese erreichen. Ein ganz besonderes Highlight waren die neuen Forschungsergebnisse von Herrn Bahr zum Thema „Seelisches Trauma“. In den Fallvorstellungen der Kollegen wurde die Bedeutung furr die Alltagspraxis sehr deutlich.

Während sich dieses Jahr die Vortragsthemen um das Metallelement rankten, wird im nächsten Jahr das Erdelement imVordergrund stehen. Die sich daraus ergebenen Themen wie Ernährung nach den Fünf Elementen mit Live-Kochen im Vortragssaal und Workshops zur umweltmedizinischen Testung von Belastungen und Austestung von Zahnmaterialien mit integriertem RAC-Training sind ein kleiner Vorgeschmack auf die Höhepunkte Timmendorf 2016.

Erfreuliches ist auch aus der Welt der Wissenschaft zu berichten. Der Nobelpreis für Medizin ging in diesem Jahr an die chinesische Professorin Tu Youyou, die als TCMExpertin in der Malariaforschung tätig ist und dabei in TCM-Rezepturen den „Einjährigen Beifuß“ als vielversprechendes Heilkraut entdeckte und erforschte. Dies zeigt, welch einen großen Schatz die Medizin mit der TCM in den Händen hält. Forscher wie auch klinisch tätige Ärzte können das Erfahrungswissen der Jahrtausende nutzen, wenn sie bereit sind,Vorbehalte abzulegen.

Dieses Heft wurde von den österreichischen Kollegen sehr engagiert und interessant gestaltet. Mögen die Artikel Ihnen Bereicherung und Hilfe für den Praxisalltag sein.

Mit den besten Wünschen für das Weihnachtsfest und ein gesundes neues Jahr!

Ihr

\section{B. Ramme}

P.S.: Wussten Sie, dass die historische Grundlage (1150 n. Ch.) für die Maßeinheit des „Scottish INCH“ die Breite des Daumens in Höhe der Nagelfalzwinkel ist [1]? CUN und INCH haben damit ursprünglich das gleiche Körpermaß.Während sich INCH zu einem Standardmaß entwickelt hat (SI units: $24.5 \mathrm{~mm}$ ), ist CUN ein individuelles Körpermaß geblieben.

[1] https://en.wikipedia.org/wiki/Inch vom 11.11.2015

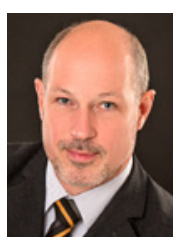

Dr. med. Bernd Ramme

1. Vorsitzender der DAA e.V.,

Osserstraße 40, D-81679 München

Tel. $+4989 / 8145252$

E-Mail des allg.Büros: kontakt@akupunktur.de, Internet:www.akupunktur.de 